\title{
Impact study of electrode and discharge in shock tube act to the shock flow
}

\author{
Shuangyan Shen ${ }^{\mathrm{a}}$, Xing Jin ${ }^{\mathrm{b}}$, Peng Zhang ${ }^{\mathrm{c}}$ \\ Equipment Academy, State Key Laboratory of Laser Propulsion \& Application, Beijin 101416, China \\ assy_fly@163.com, bjinxing_beijing@sina.com, czhangpengtf@126.com
}

Keywords: Shock tube, Corona discharge, Schlieren, Shock wave

\begin{abstract}
It received wide attention of the plasma enhance ignition. Shock tube is important equipment for the measurement of hydrocarbon fuel ignition. The schlieren system was adopt to observe the flow field in the shock tube. The influence of the discharge in low pressure section in the shock tube for incident and reflect shock wave was studied. The influence after adding three kinds of electrodes was studied. The variation of the flow field before and after the discharge was discussed. The results show that the mace type with plane end face electrode would produce bigger influence to the incident and reflect shock wave. The mace type with point end face electrode would produce little influence to the flow field and the influence of the needle type electrode could be ignored. The influence the corona discharge did to the flow field could be ignored compared with and without the discharge
\end{abstract}

\section{Introduction}

The fuel flow through the combustion chamber in supersonic speed in scramjet and the fuel is difficult to mix with air and hard to ignition and energy release in full. Plasma can not only improve the reaction temperature and radical concentration and received the widespread attention in recent years $^{[1]}$.

Shock tube is an important equipment in measuring hydrocarbon fuel ignition delay ${ }^{[2]}$. The plasma enhance hydrocarbon fuel ignition could be studied in the shock tube by adding plasma. The most typical study is did by Kosarev ${ }^{[3-5]}$. The nanosecond pulse discharge was added in the end of the shock tube. The ignition delay of $\mathrm{C} 1 \sim \mathrm{C} 5$ alkanes, ethylene and acetylene shorten by plasma was studied. Campbel1 ${ }^{[6]}$ did numerical simulation of methane/air mixture ignition adding a number of radical. The method is adding a certain concentration of $\mathrm{CH}_{3}, \mathrm{CH}_{2}, \mathrm{CH}$ and $\mathrm{C}$ radical as initial components.

The electrode should be added in the shock tube and discharge. The discharge of DC power supply would course unknown influence to the shock as the adding of electrode and discharge. The schlieren experiment system is designed to study the influence of electrode and discharge to the shock wave.

\section{Shock tube schlieren measurement system}

The shock tube schlieren measurement system diagram is shown in figure 1 .

The schlieren method is an important method to measure and record the flow. The high speed camera and schlieren optical technology could record the flow field influenced by the electrode and discharge accurately and visually.

The experiment was done in the square test section under pressure of 1.0atm. The observation window is $80 \times 60 \mathrm{~mm}$ at the square test section. The PHANTOM V711 high-speed color camera was used in high resolution mode. The high frame rate could reach more than $10000 \mathrm{fps}$ in the whole field of view. The frame rate would rise further at reducing the resolution properly in the whole field of view (1240×800 pixels). The filming resolution was set to $720 \times 400$ as the shock wave speed is very fast. The filming speed could reach 2300fps and the exposure time was set to $5 \mu \mathrm{s}$. High speed CCD camera could be external triggered by the pressure of shock or light signal. The original signal could 
be edited into TTL level to trigger the camera through DG645 synchronous controller. The delay of the camera is about $1 \mathrm{~ms}$ before the filming to ensure that the filming process could be fully recorded.

The standard of " $Z$ " glyph light path layout of schlieren system was used. The light source and imaging system was arranged to the side of the parallel light flow field test section at about $5^{\circ}$ off-axis. The shock tube test section was placed at the center of light path as shown in figure 1 . The light source of the schlieren system is high pressure xenon lamp. The xenon lamp emits the light through a $2 \mathrm{~mm}$ hole and forms the light source. A beam of parallel light was formed through a plane mirror and concave mirror. The parallel light passed through the concave mirror and plane mirror after the test section and was cut by the edge and finally shot into the camera lens. The image of the single light source is formed at the edge when the flow field is uniform in the pipeline and it makes the light evenly on the objective. An image would deviate the light source at the edge plane when the flow field density is non-uniform in the test section because the light is bending. The illumination on the camera lens would change qualitatively with the density difference. The images of the first derivative flow field distribution recorded by the high-speed camera would present the shape and location of the shock wave in the shock tube.

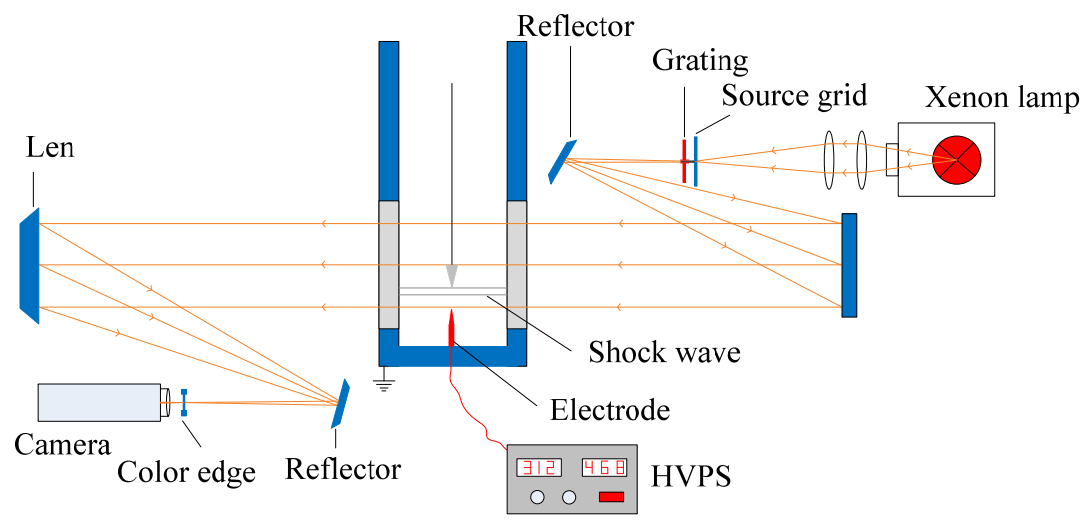

Fig. 1 Schematic diagram of the schlieren measurement system

Three kinds of discharge electrodes were designed as shown in figure 2. Figure (a) is mace type electrode and the end face is plane. Figure (b) is modified electrode in order to reduce the influence of the shock wave caused by the electrode. The end face of the electrode is designed as point rather than plane to avoid the bow shock. Figure (c) is the further modified electrode and the electrode is designed as needle type in order to reduce the influence of shock wave caused by electrode further. The corona discharge effect caused by needle type electrode is inferior to (a) and (b) type discharge electrode. Therefore the discharge effects need to be compared further.
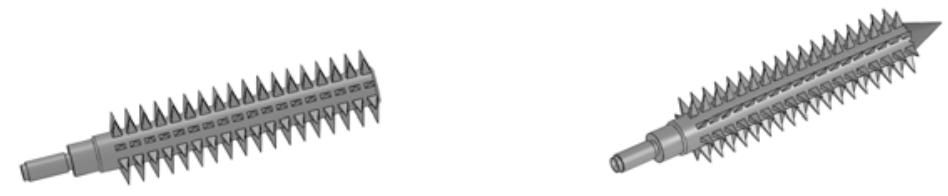

a Plane end face type electrode b Point end face type electrode

Fig. 2 Three kinds of discharge electrode

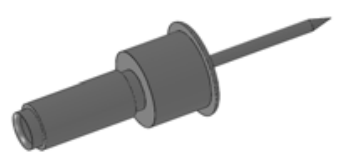

c Needle type electrode

\section{The schlieren measurement of shock tube}

The exposure time of the camera was adjust shorter to increase the shooting speed of the camera to capture the influence to the shock wave acted by the electrode and discharge. Therefore, the background would be darker compared with the images without the electrode and discharge.

The schlieren images of the mace type with plane end face are shown in figure 3 . It is shown that the incident shock wave is an obvious normal shock wave. It is normal shock wave still when it reaches the electrode. The bow shock is formed obviously outside of the electrode due to the effect of the blunt body. The reflect shock wave is normal shock wave, but the surface of the reflect shock 
wave is relative wide. The density of the gas near the electrode change obviously when the shock wave leave the electrode. The five area after the reflect shock wave is not an ideal state of zero dimension because of the effect of electrode.
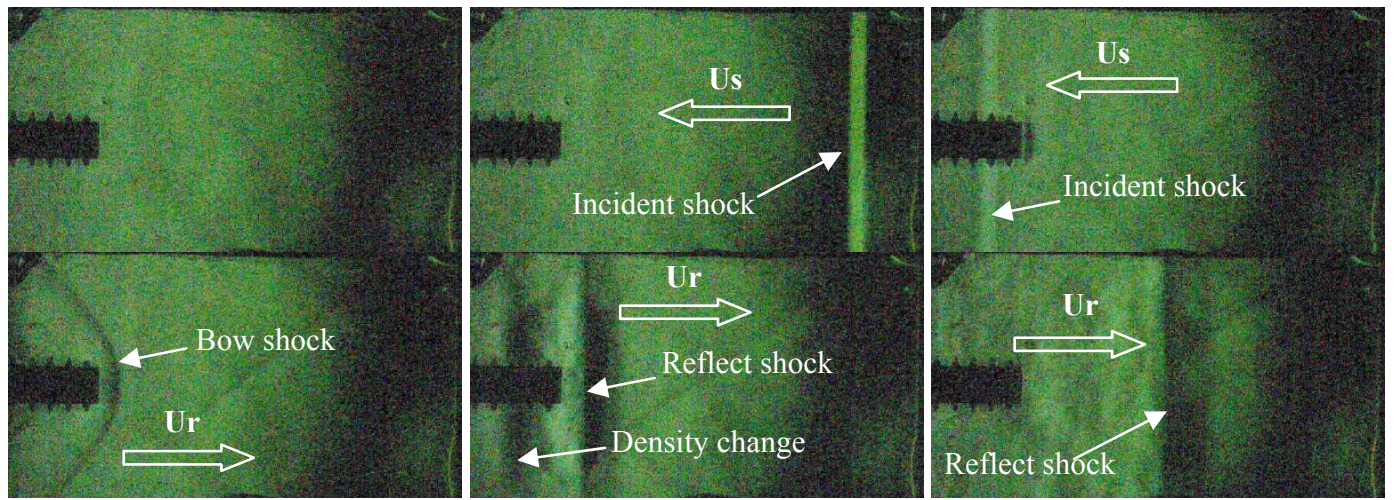

Fig. 3 The schlieren images adding mace type with plane end face electrode without discharge

The schlieren images adding mace type with point end face electrode are shown in figure 4 . It can be seen that a weaker bow shock wave was formed adding mace type with point end face electrode. The density of the gas would produce a certain change behind the reflect shock wave and near the electrode. The intensity of the reflect shock wave is stronger shown in figure 4 compared with that in figure 3. The bow shock wave is weaker caused by the point type electrode compared with the plane type electrode in comprehensive comparing with figure 3 and figure 4 . The change of the density of the air caused by electrode is less after the incident shock wave at the same time. The air flow density changes little caused by the electrode at the same time. It can be seen that the effect caused by mace type with point end face is less compared with the plane end face type.
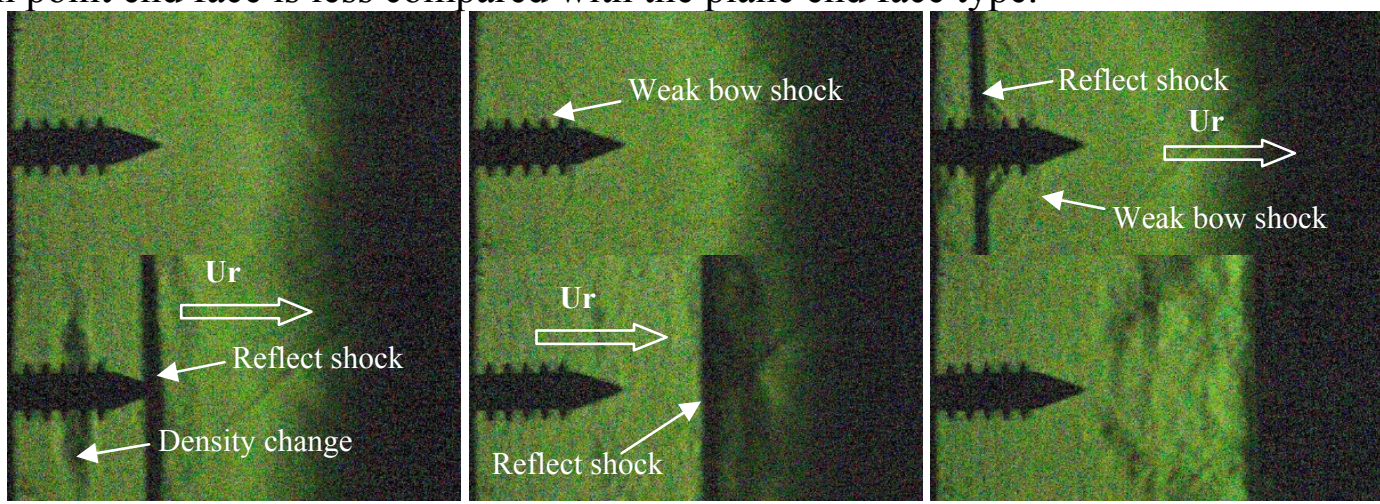

Fig. 4 The schlieren images adding mace type with point end face electrode without discharge

The schlieren is shown in figure 5 adding needle type electrode. The needle type electrode is thin and the adding of this electrode does not produce bow shock wave. The gas density changes behind the reflect shock wave and near the surface of the electrode. However, the change is small variety. Therefore, needle type electrode cause minimal impact to the shock wave.
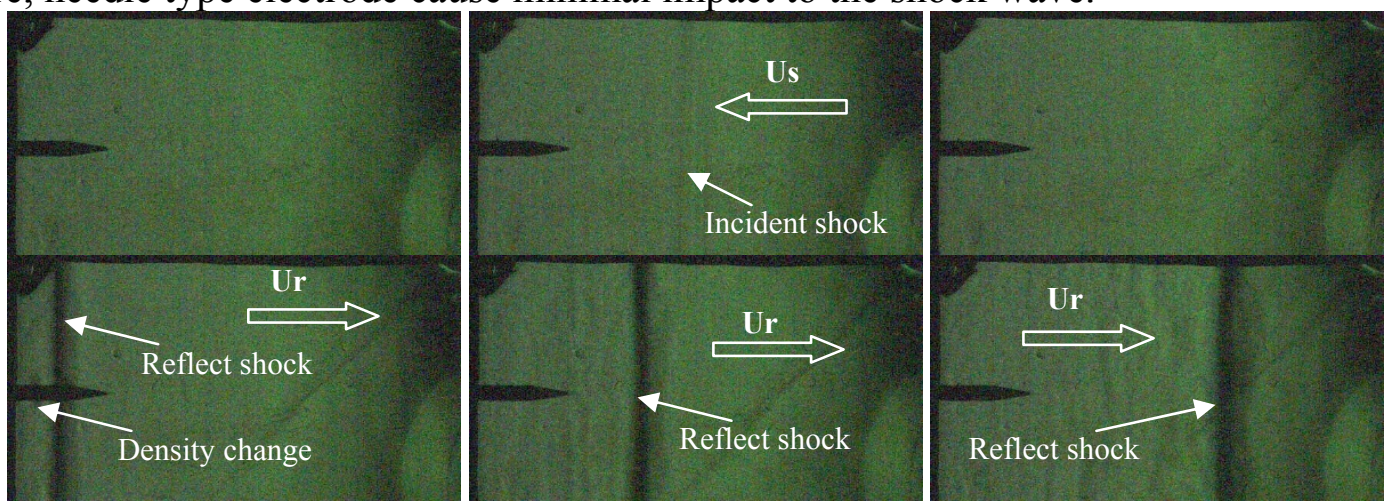

Fig. 5 The schlieren images adding needle type electrode without discharge

The schlieren images of corona discharge are shown in figure 6 adding mace electrode with plane end face in the discharge cell. The discharge voltage is $0.7 \mathrm{kV}$ and the discharge current is $5.6 \mathrm{~mA}$. The 
corona discharge did little difference to the incidence and reflects shock wave compared with figure 6 . However, the discharge cause a certain influence to the flow field after the reflect shock wave and increases the turbulence of the density.
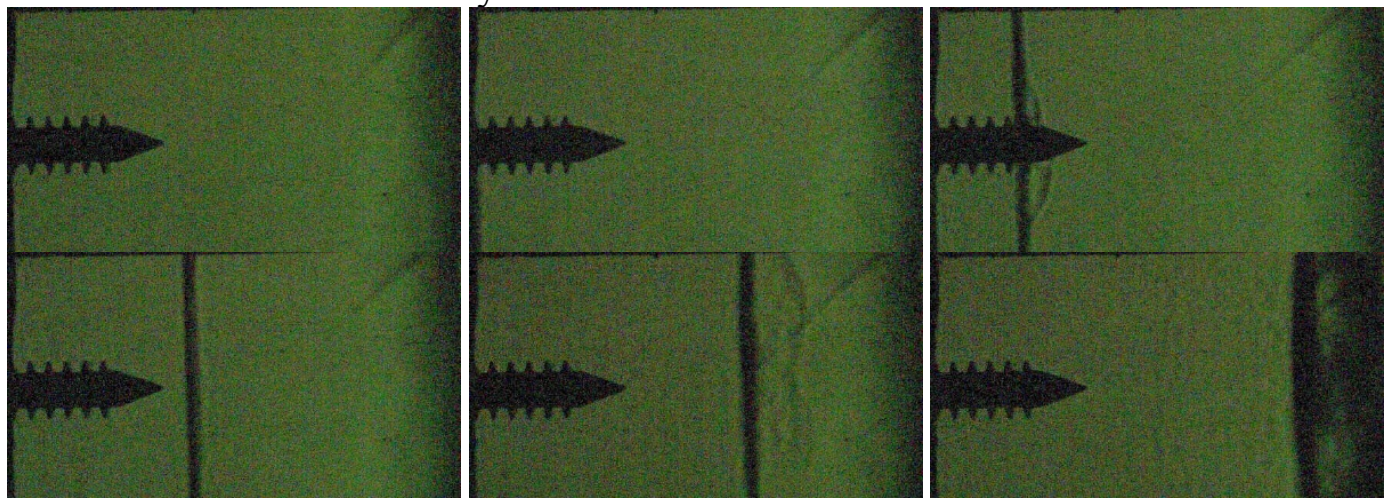

Fig. 6 The schlieren images adding mace type with point end face electrode with discharge

\section{Summary}

Three types of discharge electrodes were designed and the schlieren experiments were studied with the corona discharge in the shock tube test section. The following conclusions were got as follows:

(1) The mace type electrode with plane end face would add influence to the incidence shock wave without the discharge and the obvious bow shock wave would produce. The incidence and reflect shock wave would be broken. The bow shock wave is very weak caused by the mace type with point end face electrode. However, the gas density after the reflect shock wave would cause a certain change. There is almost no influence to the incidence shock wave and the density of the reflect shock wave change very weak when adding needle type electrode.

(2) The energy injected into the end of the shock tube through the three electrodes is small and usually in watt magnitude when the corona discharge in the low pressure section of the shock tube.

(3) The effect of the corona discharge is small for the flow process. The corona discharge slightly increase the density change of flow field after the reflect shock wave for the mace type with plane end face electrode. The effect of the corona discharge did to the flow field could basically to be ignored for mace type with point end face electrode and needle type electrode.

\section{References}

[1] S.M. Starikovskaia, Plasma Assisted Ignition and Combustion, Journal of Physics D: Applied Physics, 39(16) (2006) 265-299.

[2] G.A. Pang, D.F. Davidson, R.K. Hanson, Experimental Study and Modeling of Shock Tube Ignition Delay Times for Hydrogen- Oxygen-Argon mixtures at Low Temperatures, Proceedings of the Combustion Institute, 32(1) (2009) 181-188.

[3] I.N. Kosarev, S.V. Aleksandrov, S.M. Starikovskaia, et al, Kinetics of ignition of saturated hydrocarbons by nonequilibrium plasma: $\mathrm{CH} 4$-containing mixtures, Combustion and Flame, 154 (2008) 569-586.

[4] I.N. Kosarev, S.V. Aleksandrov, S.M. Starikovskaia, et al, Kinetics of ignition of saturated hydrocarbons by nonequilibrium plasma: $\mathrm{C} 2 \mathrm{H} 6-$ to $\mathrm{C} 5 \mathrm{H} 12-$ containing mixtures, Combustion and Flame, 156 (2009) 221-233.

[5] I.N. Kosarev, A.I. Pakhomov, S.V. Kindysheva, et al, Nanosecond discharge ignition in acetylene-containing mixtures, Plasma Sources Science and Technology, 22 (2013) 1-9. 
[6] C.S. Campbell, F.N. Egolfopoulos, Kinetics Paths to Radical-Induced Ignition of Methane/Air Mixtures, Combustion Science and Technology, 177(12) (2005) 2275-2298. 